\title{
Characteristics of Needle-Disk Electrodes Atmospheric Pressure Discharges Applied to Modify PET Wettability
}

\author{
Milton Eiji Kayama, Member, IEEE, Lucas José da Silva, Vadym Prysiazhnyi, \\ Konstantin G. Kostov, and Mauricio Antonio Algatti
}

\begin{abstract}
In the atmospheric pressure discharge with arrangement of a syringe needle, a capillary, and an external disk, it was observed different operation modes according to the relative position of the electrodes. With external electrode over the shaft of the needle, a stable and steady plume is formed from the tip of the needle to the exterior of the capillary. The breakdown is governed by filamentary discharges that evolve randomly on time in the gap between the needle and the capillary. This mechanism changes to bullet-like discharge when the external disk is located off needle. The breakdown generates a large amount of charge that rises quickly and varies with the distance between the electrodes. The mean power is higher than that of the previous case and the plume generated, in interaction with external metallic surface, is more unstable to arc transition. Independently of the operation mode, high electric field at the bevel of the needle plays an important role on the evolution of the plume. The application of the plume to change the wettability properties of the polyethylene terephthalate indicated rapid augment of the hydrophilicy and treated area larger than the plume dimension.
\end{abstract}

Index Terms-Arc discharges, atmospheric pressure plasmas, breakdown voltage, glow discharges, hydrophilic, plasma devices, polyethylene terephthalate (PET), wettability.

\section{INTRODUCTION}

$\mathbf{N}$ ONEQUILIBRIUM plasma jets that are generated by gaseous discharges at atmospheric pressure have found its applications in many fields of engineering and medical sciences. Various arrangements with wire tips, electrodes and dielectrics can be used to produce such jets, ignited and maintained by ac-signals with frequencies up to megahertz [1]. In this, a big family of devices designed to produce small plasmas have deserved special attention once the electron temperature tends to be higher than in the counterpart devices [2], [3]. The high capacity to dissociate molecules due the highpower concentration of micro plasmas makes them adequate to produce reactive radical species used to modify surfaces or dissociate molecules for plasma depositions [3]-[5]. The discharge stability and low operation voltages make them adequate for use in bio-applications [6]. The further reduction of consumed power can be achieved by using a pulsed power

Manuscript received May 24, 2016; revised December 8, 2016; accepted February 23, 2017. Date of publication April 3, 2017; date of current version May 8, 2017. This work was supported in part by FAPESP, CNPq, and CAPES

The authors are with the Faculty of Engineering, Sao Paulo State University, SP 12516-410 Guaratingueta, Brazil (e-mail: kayama@feg.unesp.br).

Color versions of one or more of the figures in this paper are available online at http://ieeexplore.ieee.org.

Digital Object Identifier 10.1109/TPS.2017.2684622 supply while keeping the same amount of exited species [7]. The device present here was developed to material surface treatment and atmospheric film deposition.

In spite of excellent bulk physical properties the polymers have poor surface properties for applications like for instance in biological environments. In particular, to change surface wettability and adhesion properties the plasma assisted processes is a known to effectively achieve these goals. The use of nonthermal plasmas to change wettability of polyethylene terephthalate (PET) have been verified by several arrangements and discharge configurations such as parallel plates dielectric-barrier-discharge (DBD) [8], ion implantation [9], dc glow discharge [10], and coaxial cylindrical array of electrodes and dielectric [11]. All these methods reduces the contact angle to about $35^{\circ}$ with treatment time less than $20 \mathrm{~s}$, except for dc glow with time in order of $10 \mathrm{~min}$. In this paper, we report the treatment using plumes produced by needle achieving similar results. It represents an advantage face to the lower voltage, low gas flow and the relative wide area of treatment compared to the plume dimensions.

The micro discharge can be generated by a simple conductor tip at atmospheric pressure [8]. The electrode can be made coaxial to some dielectric capillary allowing the gas flow axially. That gives protection to users once direct contact is avoided. Surface charges tend to be adsorbed at interior surface and affect the initiation of the discharge [9], [10]. Fast framing photograph shows that with sinusoidal excitation in $\mathrm{kHz}$ frequencies or square pulses the discharge evolves with ionization fronts in form of bullets propagating axially at speeds in the range of $10^{4}-10^{5} \mathrm{~m} / \mathrm{s}$ [11]-[13]. The plume length is proportional to the signal frequency and the applied voltage and either high or low gas flow reduces the plume extension [14]. An external coaxial electrode can also be incorporated. At downstream position, analysis of captured images shows that it reduces the plume length but increases its diameter [15], [16]. Due to small dimensions, diagnostics of such plasmas is a difficult task. Electron density is estimated to be around $10^{11}-10^{14} \mathrm{~cm}^{-3}$ obtained from Stark linebroadening data analysis [17], [18] and gas temperature $T_{g}$ typically ranges from 300 to $350 \mathrm{~K}$ estimated by emission spectroscopy [11], [19]. An extended discussion on this topic can be found elsewhere [20].

In this paper, we describe the study of an arrangement of electrodes with a needle and a thin disk electrode movable in any position along a capillary between these electrodes. 

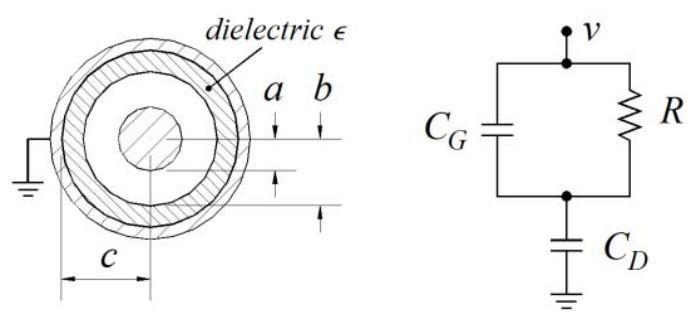

Fig. 1. Cylindrical discharge and equivalent circuit.

The mechanism that leads to the evolution of the discharge is governed either by sequences of micro filamentary discharges or fast ionization fronts leading to a large multiplication of charges. The mean power at breakdown is higher in the latter case. The plume formed from high-intensity electric field at needle tip extends downstream of the capillary. Once in interaction with a metallic surface it can change to arc regime or maintain a steady regime depending on the mechanism used to create it. The details of these investigations are shown in forward sections.

\section{Discharge Circuit Analysis}

The equivalent circuit of the discharge with cylindrical arrangement of coaxial conductors and dielectric is shown in Fig. 1. The impedance of the circuit is given by

$$
Z=\frac{R}{1+\left(\omega R C_{G}\right)^{2}}-j \frac{1+\left(\omega R C_{G}\right)^{2}+C_{D} C_{G}(\omega R)^{2}}{\omega C_{D}\left(1+\omega R C_{G}\right)^{2}}
$$

where $C_{G}$ and $C_{D}$ are the gap and dielelectric capacitances, respectively. The active power $P=V^{2} \operatorname{Re}[Z] /\left(2|Z|^{2}\right)$ is

$$
P=\frac{V^{2} \omega^{2} R C_{D}^{2}}{2\left[1+\omega^{2} R^{2}\left(C_{G}+C_{D}\right)^{2}\right]} .
$$

For needle-disk like and even for parallel plate arrangements, the second term in denominator is negligible in comparison to the first one. So, the active power can be reduced to

$$
P=\frac{V^{2} \omega^{2} R C_{D}^{2}}{2} \text {. }
$$

For a cylindrical case, neglecting fringe effects in discharge region, we have $C_{D}=\epsilon \epsilon_{0} 2 \pi h / \ln (c / b)$ and $R=\eta \ln (b / a) /(2 \pi h)$. Defining $\xi=b / c$ and $\beta=a / c$ the normalized power $p=P /\left(h V^{2} \omega^{2} \epsilon_{0}^{2} \eta\right)$ can be written in the form

$$
p=\pi \epsilon^{2} \frac{\ln \left(\frac{\xi}{\beta}\right)}{(\ln \xi)^{2}} .
$$

Therefore in cylindrical arrangements the power is maximized with large gap and a thin dielectric with high dielectric constant.

\section{EXPERIMENT}

The capillary electrode was a stainless steel syringe needle with $0.7 \mathrm{~mm}$ outer diameter. A jacket of borosilicate capillary with $1.0 \mathrm{~mm}$ inner and $1.5 \mathrm{~mm}$ outer diameter evolves the needle extending $10 \mathrm{~mm}$ from its tip. The needle was used in its original conditions except for the end opposite to the

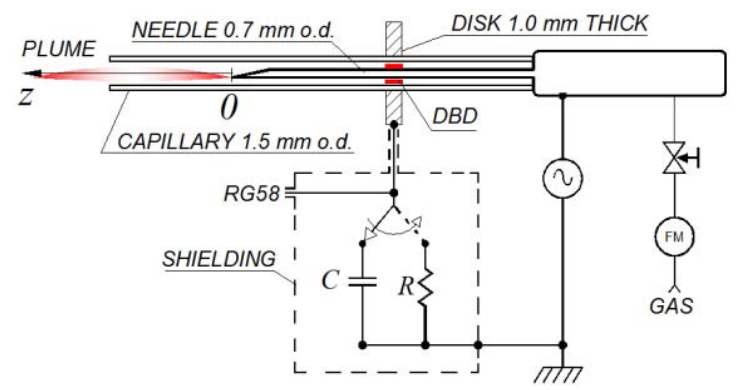

Fig. 2. Diagram of the experimental setup.

tip that was adapted for gas injection. A brass disk, $1.0 \mathrm{~mm}$ tick with an orifice of $1.6 \mathrm{~mm}$ diameter, coaxial to the arrangement completes the set. The gas flows axially through the needle fed by gas system with flow control. Sinusoidal signal was applied between the needle and the disk using a power supply Minipulse 6 (GBS Elektronik GmbH, Germany). The frequency used was $37 \mathrm{kHz}$ with maximum peak-topeak voltage around $5.5 \mathrm{kV}$. The limit of voltage is due to overheating of borosilicate at the discharge site that results in the glass cracking. Ar gas flow was used as a media to produce plasma and gas flow was fixed to $0.5 \mathrm{~L} / \mathrm{min}$. The disk was connected to the ground through either capacitor or resistor and mounted in a metallic box and wrapped by aluminum foils and connected to Tektronix oscilloscope with RG-58 coaxial cable. A Tektronix 1000X probe connected right over the needle base was used to measure the applied voltage. The diagram shown in Fig. 2 summarizes the experimental setup. The treatment of PET was made on foils $0.1 \mathrm{~mm}$ thick over grounded aluminum disk $75-\mathrm{mm}$ diameter and $5 \mathrm{~mm}$ tick, at $7 \mathrm{~mm}$ from the capillary end.

\section{RESULTS AND DISCUSSION}

\section{A. Breakdown of Argon}

The current signals for the discharge were similar to those found in other experiments with ground electrode located over the needle and at downstream position [12], [16], [21]. A slight difference for the present arrangement will be commented later on. In order to detect the early signals on the threshold of breakdown the applied voltage was risen manually varying the bridge voltage in the power supply. The capacitor was connected between the external disk electrode and the ground to accumulate the charge. A high capacitance was used in order to generate a ringing signal to trigger the oscilloscope in normal/single-shot mode. This ringing arose from the charge exchange between reactive stray elements in the circuit. Two distinct waveforms on the capacitor were observed depending on the axial position of the external electrode.

Let us consider $z$-axis on the axis of capillaries in the direction of the gas flow to the exit with $z=0$ at its tip of the needle. With the external disk located over the shaft $(z<0)$, the discharge operates as a cylindrical DBD, showing a presence of multispikes on the capacitor voltage signal. Those spikes are related to filamentary discharge that evolves randomly in the volume between two electrodes influenced by surface charges at the dielectric. The signals are close 


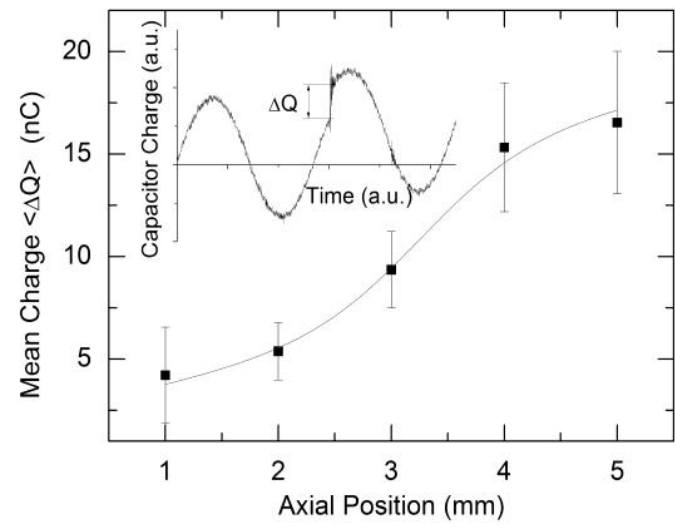

Fig. 3. Charge generated at breakdown in Ar.

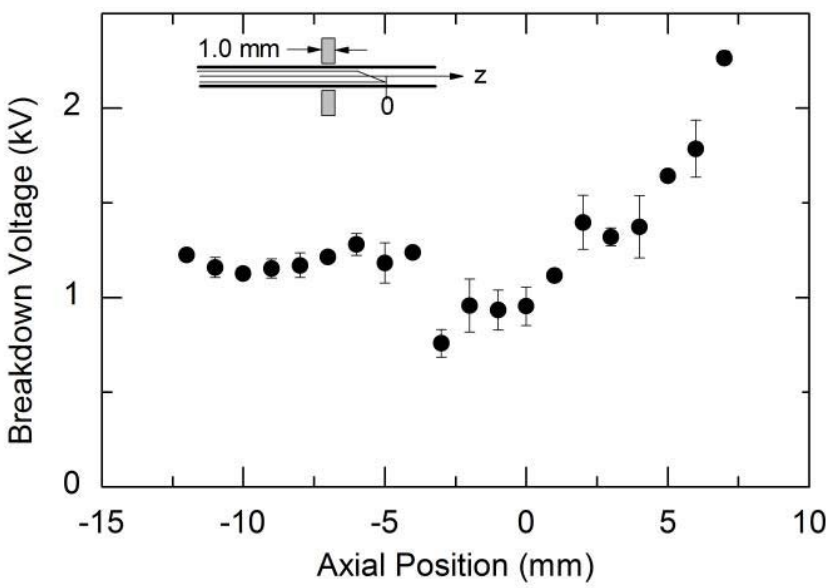

Fig. 4. Breakdown voltage in Ar with relative position of cathode with the needle tip.

to that observed in similar experiment in [21]. Moving the external electrode to the bevel and at downstream in $z>0$ locations the signals show a fast rise on charge by $\Delta Q$ as consequence of the breakdown in the gap. The major part occurs during positive voltage half cycle at ratio of around $7: 3$ over negative voltage. The mean value of charge $\langle\Delta Q\rangle$ evaluated over at least three signals is shown in Fig. 3. The charge rises proportionally to the separation electrodes varying from about 5 to $15 \mathrm{nC}$ in 5.0-mm interval. That corresponds to a multiplication factor of 1.2-2.2 to the initial charge promoted by the ionization on the gap.

In spite of negligible variation on the applied voltage during the recorded period of time, the breakdown evolves differently according to the position of the external electrode. The results are shown in Fig. 4. For $z<0$, the spikes on the signal are randomly distributed. For $z>0$, the rising on the signal might repeat or not in sequential half cycles. In case of one single breakdown event the signal remains modulated to single pulse originated from the discharge of stray capacitances on a large resistance associated with the absence of charged species in the gap. That behavior was simulated using arrangement of capacitors in parallel to a single pulse power source [21], [22]. The arrangement was two capacitances in series corresponding to the experimentally measured capacitance in absence of

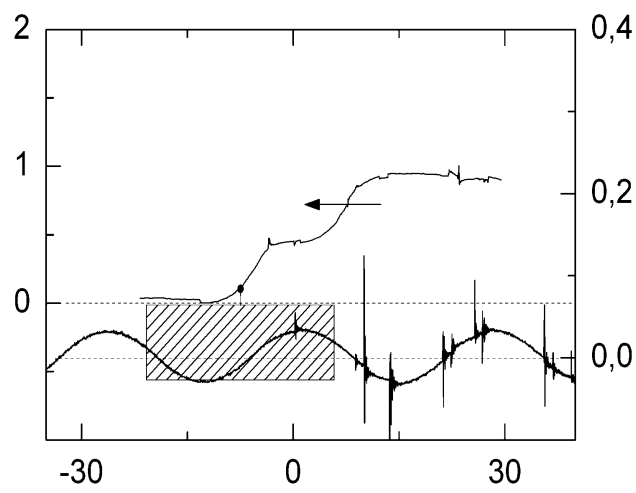

(a)

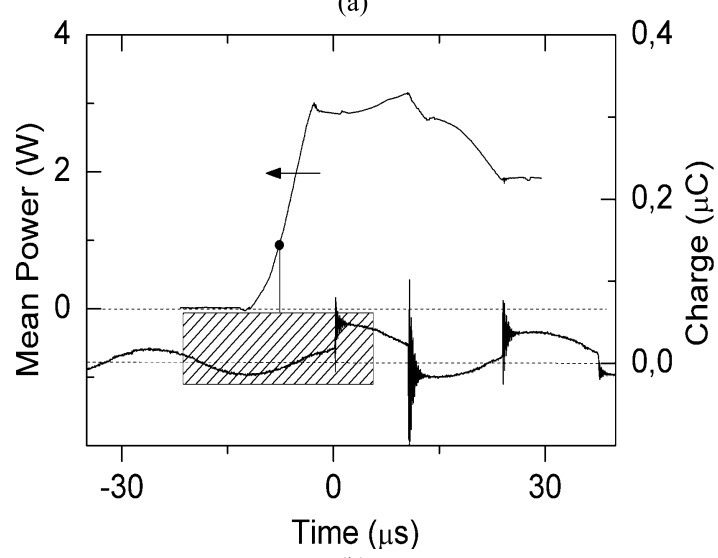

(b)

Fig. 5. Mean power on early phase of discharge with disk (a) over the shaft of the needle and (b) at downstream, calculated at time $t$ between $t-T / 2$ and $t+T / 2$ shown by shaded area on charge signal.

plasma and of the capacitor used for charge measurement. Once charged by the single pulse the voltage rises and starts to discharge with a characteristic time greater than the period of the signal. Modulated to a sinusoidal power source the resulting waveform is similar to that observed in the experiment. This suggests that the breakdown mechanism promotes a fast charge multiplication followed by its extinction leaving a poorly ionized gas between the electrodes. The actual ionization level in the preionized gas determines the occurrence of breakdown in the following half cycles of applied voltage.

The mean power during a cycle in this transition of the gap to conduction state was calculated using the area of Lissajous figures in graphics of charge versus applied voltage [23], [24]. Fig. 5 shows the evolution of mean power with occurrence of breakdown. The power rises at first event due to charge accumulation as a result of ionization of gas at the electrodes gap. The applied voltage is on the threshold of breakdown which makes the event being randomly either extinguished or repeated in sequential half-cycle. The extinction leads to a continuous decay on the accumulated energy. The repetition of the event leads to the rise on the power which represents generation of additional charges. The progressive rising of the power indicates that a net charge is continuously produced after the breakdown. The power varies according to external electrode location. When it is located over the needle shaft $(z<0)$ the power was approximately $0.5 \mathrm{~W}$ or less. Moving the electrode downstream $(z>0)$ leads to increased power generation to 
about 3.0 W. There were no clear dependence between the mean power and the electrode position for the first breakdown.

The breakdown was detected by variation on the charge and/or the augment of the mean power. The variation of the mean value of voltage during this event, using at least three signals, with the position of external electrode along the capillary is shown in Fig. 4. The breakdown voltage remains around $1.2 \mathrm{kV}$ for the disk over the shaft with small variation due to local misalignment on the arrangements of electrodes and dielectric capillary. But at position $z \approx-3 \mathrm{~mm}$, the breakdown voltage rapidly drops to about $0.9 \mathrm{kV}$. The high electric field associated with this border is the cause for the reduced breakdown voltage for the disk over the bevel. With a disk outside further away from the needle the breakdown voltage rises in proportion to the distance to the tip up to $2 \mathrm{kV}$ at $z=6 \mathrm{~mm}$ of distance. The increase on that voltage leads to the rise on electron mean energy and to the ionization process.

\section{B. Continuous Operation}

On increasing the voltage over the breakdown, the discharge evolves in the capillary. The arrangement of the syringe needle and the external electrode grounded and at downstream location is characterized by propagation of bullets and the formation of jet that extends to the exterior of the capillary [15], [16], [25]. Other arrangement uses the external electrode over the needle shaft with similar formation of plasma jet [21]. In our experiment, we have observed two different behaviors of the plume when interacting with a grounded metallic plate at the exit of the capillary. With the external disk at downstream location a bright plume is formed. For a short distance, arc-like transition occurs, forming visually stable and bright filament, which is actually a sequence of filament reappearing each period at the same place. Positioning the disk over the needle shaft results to plume brightness decrease, but making it not sensitive to variations, and changing the discharge regime while interacting with the plate. Considering that the desired application of the device is for surface treatment, we investigate this operational mode.

With external disk electrode at $z=-10 \mathrm{~mm}$, a steady plume is formed within $0.4-1.2 \mathrm{~L} / \mathrm{min}$ range of Ar flow. It was fundamental to keep the original profile of the needle bevel. Making it flat led to the suppression of the plume. The limiting peak voltage to preserve the capillary was about $5.5 \mathrm{kV}$. The variation of power is in the range of $30-800 \mathrm{~mW}$. The raw current signal showing the displacement current superposed to the DBD current measures on $380-\Omega$ resistance is shown in Fig. 6 with a typical picture of the plume.

The length of the plume created by ionization wave varies according to the gas flow rate and applied voltage. It is known that the plume does not extend too far from the exit of capillary due to the gas dynamic effect and interaction with the surrounding ambient. The length here was taken from grayscale variation of the pictures. As shown in Fig. 7, the plume length is proportional to the mean power of the discharge at flow rate under around $1.0 \mathrm{~L} / \mathrm{min}$. For Ar the plume extinguishes at flow over this value.

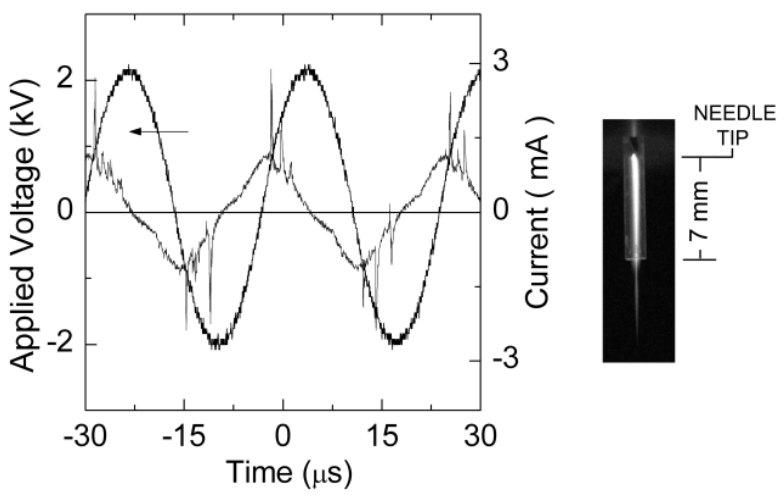

Fig. 6. Left: raw signals of applied voltage and current. Right: picture of plasma plume.

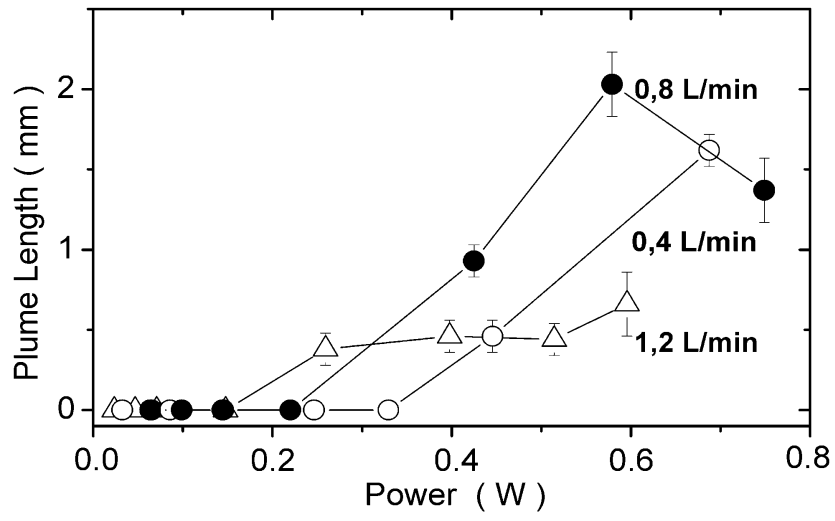

Fig. 7. Dependence of the Ar plume length with applied power for various flow rate.

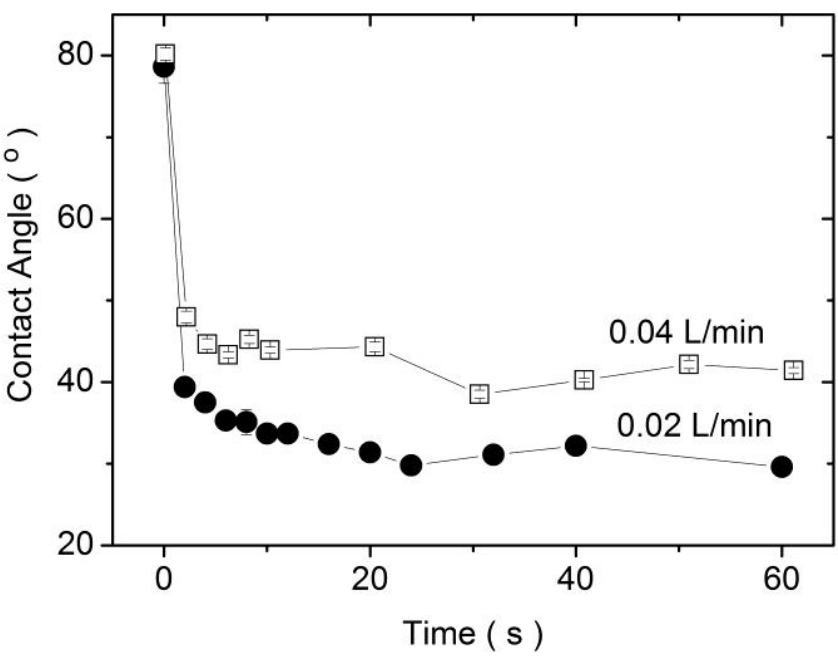

Fig. 8. Variation of contact angle with the time of plasma interaction.

\section{PET Treatment}

The hydrophilic analysis of the PET with static $0.2-\mathrm{mm}$ diameter drop performed at attack point of the plume indicate the formation of polar groups with plasma-surface interaction less than $10 \mathrm{~s}$ (Fig. 8). The contact angle varies from about $78^{\circ}$ to saturate around $30^{\circ}$ after $20 \mathrm{~s}$ of interaction. That behavior was similar to flow rate at 0.2 and $0.4 \mathrm{~L} / \mathrm{min}$. The dimension of the plume in interaction with the surface remains the same keeping close plasma parameters as well. The contact angle 


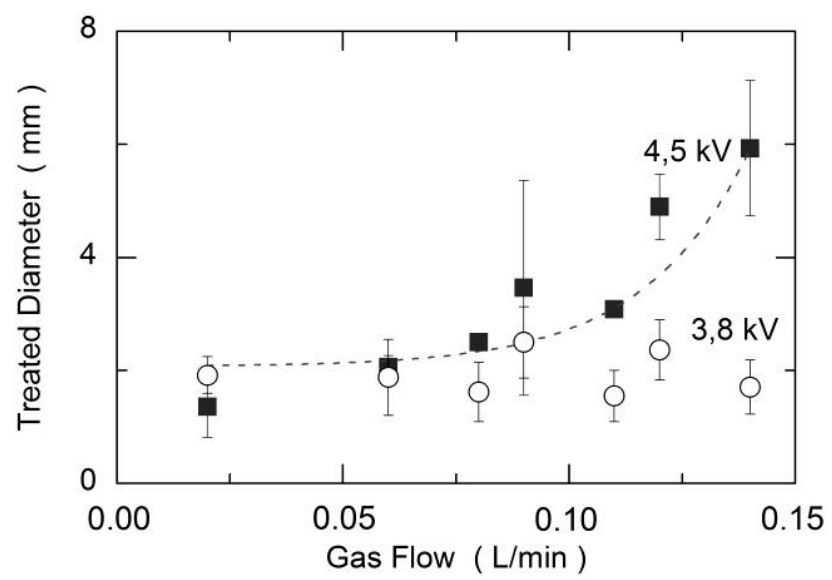

Fig. 9. Variation of treated diameter with gas flow and applied voltage.

around the plasma-surface interaction center indicated a wider treated area due to gas dynamic effect. In order to stablish a dimension of the treated region the radial variation of the contact angle was fit to Gaussian and Lorentzian profiles, respectively, in the form of $\theta=\theta_{1}+\theta_{2} \exp \left[-4 \ln 2\left(\left(r-r_{0}\right) / \Delta R\right)^{2}\right]$ and $\theta=\theta_{3}+\left(2 \theta_{4} / \pi\right) \Delta R /\left(4\left(r-r_{0}\right)^{2}+\Delta R^{2}\right)$ where $v_{1}, v_{2}$, $v_{3}$, and $v_{4}$ are adjusting parameters, $r$ the radial coordinate, $r_{0}$ the radial shift and $\Delta R$ the full width at half height of the curve. This last parameter was considered as diameter of the circle of treated area. The dependence of this diameter with the discharge power is shown in Fig. 9. At $3.3 \mathrm{kV}$ the treated diameter is about $1.8 \pm 0.5 \mathrm{~mm}$ insensitive to the gas flow. At higher voltage, $4.5 \mathrm{kV}$, it reaches $4 \pm 1 \mathrm{~mm}$ at high flow rate. That might be associated with reduction of the interaction of the plume with surrounding gas after the exit the capillary.

\section{CONCLUSION}

The characteristics of micro plasma device made of an arrangement of a syringe needle, disk and capillary were investigated under excitation of sinusoidal waveform at $37 \mathrm{kHz}$. On the threshold of the breakdown, it was observed a different evolution of the discharge according to the relative position of the disk with the needle bevel. With the disk over the shaft, on the threshold of breakdown, the discharge evolves with random micro discharges that show up in form of short spikes. This is consistent with filamentary DBD discharges that evolves in the gap between the capillary and the needle. With external disk out of needle zone, the charge showed a fast rise consistent with bullet propagation observed in this arrangement of electrodes. This charge and breakdown voltage increases proportionally to the separation between the electrodes. The breakdown voltage is low with disk over the bevel due to the intense electric field at knife edges and quasi constant over the shaft where the electric field is uniform. The intense electric field at needle tip was observed to be fundamental to generate plasma plume particularly for the disk over the shaft of the needle. The plume evolves with appropriate control of applied voltage and gas flow, and is inhibited when in interaction with ambient gas outside the capillary. In interaction with a grounded metallic surface, the plume is more stable to arc-like transition when excited by DBD discharge with the disk over the shaft of the needle. The length of the plume increases with the mean power of this discharge and tends to reduce at high gas flow due to change of regime flow.

The application of the plume on the change of wettability of PET surfaces has shown rapid change on the hydrophilicy and treated area larger than the visible plasma-surface interaction dimension. That was achieved at higher voltage and gas flow rate.

\section{ACKNOWLEDGMENT}

The authors would like to thank J. B. Galhardo for technical support.

\section{REFERENCES}

[1] X. Lu, M. Laroussi, and V. Puech, "On atmospheric-pressure nonequilibrium plasma jets and plasma bullets," Plasma Sour. Sci. Technol., vol. 21, no. 3, p. 034005, Apr. 2012.

[2] P. Bruggeman and R. Brandenburg, "Atmospheric pressure discharge filaments and microplasmas: Physics, chemistry and diagnostics," J. Phys. D, Appl. Phys, vol. 46, no. 46, p. 464001, 2013.

[3] D. Mariotti and R. M. Sankaran, "Microplasmas for nanomaterials synthesis,” J. Phys. D, Appl. Phys., vol. 43, no. 32, p. 323001, Jul. 2010.

[4] E. E. Kunhardt, "Generation of large-volume, atmospheric-pressure, nonequilibrium plasmas," IEEE Trans. Plasma Sci., vol. 28, no. 1, pp. 189-200, Feb. 2000.

[5] K. H. Becker, K. H. Schoenbach, and J. G. Eden, "Microplasmas and applications," J. Phys. D, Appl. Phys., vol. 36, no. 3, p. R55, 2006.

[6] X. Lu et al., "A simple atmospheric pressure room-temperature air plasma needle device for biomedical applications," Appl. Phys. Lett., vol. 95, no. 18, p. 181501, 2009.

[7] J. L. Walsh, J. J. Shi, and M. G. Kong, "Contrasting characteristics of pulsed and sinusoidal cold atmospheric plasma jets," Appl. Phys. Lett., vol. 88, no. 17, p. 171501, 2006.

[8] C. Huang, Y.-C. Chang, and S.-Y. Wu, "Contact angle analysis of low-temperature cyclonic atmospheric pressure plasma modified polyethylene terephthalate," Thin Solid Film, vol. 518, no. 13, pp. 3575-3580, Apr. 2010.

[9] Z. Fang, J. Yang, Y. Liu, T. Shao, and C. Zhang, "Surface treatment of polyethylene terephthalate to improving hydrophilicity using atmospheric pressure plasma jet," IEEE Trans. Plasma Sci., vol. 41, no. 6, pp. 1627-1634, Jun. 2013.

[10] K. N. Pandiyaraj, V. Selvarajan, R. R. Deshmukh, and G. Gao, "Adhesive properties of polypropylene (PP) and polyethylene terephthalate (PET) film surfaces treated by DC glow discharge plasma," Vacuum, vol. 83, no. 2, pp. 332-339, Sep. 2009.

[11] C. Gong, X. Tian, S. Yang, R. K. Y. Fu, and P. K. Chu, "Surface treatment of polyethylene terephthalate using plasma ion implantation based on direct coupling of RF and high-voltage pulse," IEEE Trans. Plasma Sci., vol. 40, no. 2, pp. 487-491, Feb. 2012.

[12] H. Koinuma et al., "Development and application of a microbeam plasma generator," Appl. Phys. Lett., vol. 60, no. 7, pp. 816-817, 1992.

[13] T. Gerling, T. Hoder, R. Brandenburg, R. Bussiahn, and K.-D. Weltmann, "Influence of the capillary on the ignition of the transient spark discharge," J. Phys. D, Appl. Phys, vol. 46, no. 14, p. 145205, 2013.

[14] Y. B. Golubovskii, V. A. Maiorov, J. F. Behnke, J. Tepper, and M. Lindmayer, "Study of the homogeneous glow-like discharge in nitrogen at atmospheric pressure," J. Phys. D, Appl. Phys., vol. 37, no. 9, p. 1346, 2004.

[15] A. Sarani, C. Nicula, X. F. Gonzales, and M. Thiyagarajan, "Characterization of kilohertz-ignited nonthermal $\mathrm{He}$ and $\mathrm{He} / \mathrm{O}_{2}$ plasma pencil for biomedical applications," IEEE Trans. Plasma Sci., vol. 42, no. 10, pp. 3148-3160, Oct. 2014.

[16] C. Jiang et al., "Single-electrode He microplasma jets driven by nanosecond voltage pulses," J. Appl. Phys., vol. 119, no. 8, p. 083301, 2016.

[17] J. Shi, F. Zhong, J. Zhang, D. W. Liu, and M. G. Kong, "A hypersonic plasma bullet train traveling in an atmospheric dielectric-barrier discharge jet," Phys. Plasmas, vol. 15, no. 1, p. 013504, Jan. 2008.

[18] L. Chen, P. Zhao, X. Shu, J. Shen, and Y. Meng, "On the mechanism of atmospheric pressure plasma plume," Phys. Plasmas, vol. 17, no. 8, p. $083502,2010$. 
[19] G. Cho et al., "Cold plasma jets made of a syringe needle covered with a glass tube," IEEE Trans. Plasma Sci., vol. 39, no. 5, pp. 1234-1238, May 2011.

[20] Z. Cao et al., "Spatially extended atmospheric plasma arrays," Plasma Sour. Sci. Technol., vol. 19, no. 2, p. 025003, 2010.

[21] J.-S. Oh, J. L. Walsh, and J. W. Bradley, "Plasma bullet current measurements in a free-stream helium capillary jet," Plasma Sour. Sci. Technol., vol. 21, no. 3, p. 034020, 2012.

[22] S. Hübner, S. Hofmann, E. M. van Veldhuizen, and P. J. Bruggeman, "Electron densities and energies of a guided argon streamer in argon and air environments," Plasma Sour. Sci. Technol., vol. 22, no. 6, p. 165011, 2013.

[23] A. Shashurin and M. Keidar, "Experimental approaches for studying non-equilibrium atmospheric plasma jets," Phys. Plasmas, vol. 22, no. 12, p. 122002, 2015.

[24] A. P. Papadakis, S. Rossides, and A. C. Metaxas, "Microplasmas: A review," Open Appl. Phys. J., vol. 4, pp. 45-63, Dec. 2011.

[25] Y. Hong et al., "Low temperature air plasma jet generated by syringe needle-ring electrodes dielectric barrier discharge at atmospheric pressure," Thin Solid Films, vol. 548, pp. 470-474, Dec. 2013.

[26] S. Liu and M. Neiger, "Excitation of dielectric barrier discharges by unipolar submicrosecond square pulses," J. Phys. D, Appl. Phys., vol. 34, no. 11, p. 1632,2011

[27] J. Kriegseis, B. Möller, S. Grundmann, and C. Tropea, "Capacitance and power consumption quantification of dielectric barrier discharge (DBD) plasma actuators," J. Electrostatics, vol. 69, no. 4, pp. 302-312, Aug. 2011.

[28] D. E. Ashpis, C. L. Laun, and E. L. Griebeler, "Progress toward accurate measurements of power consumptions of DBD plasma actuators," Tech. Rep. NASA/TM-2012-217449, 2012. NASA, Cleveland, OH, USA. [Online]. Available: http://www.sti.nasa.gov

[29] A. Sobota, O. Guaitella, and A. Rousseau, "The influence of the geometry and electrical characteristics on the formation of the atmospheric pressure plasma jet," Plasma Sour. Sci. Technol., vol. 23, no. 2, p. $025016,2014$.

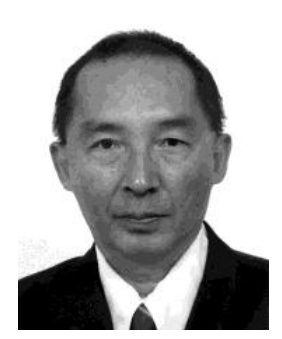

Milton Eiji Kayama (M'10) received the B.S. degree in physics and the Ph.D. degree in electrical engineering from the State University of Campinas, Campinas, Brazil, in 1987.

From 1988 to 1990 , he was a Visiting Professor at the University of Washington, Seattle, WA, USA. Since 1990, he has been a Professor at Sao Paulo State University, Guaratingueta, Brazil. He teaches in the field of electromagnetism and plasma physics. His current research interests include plasma generation, high-voltage engineering and diagnostics of hot plasmas with Thomson scattering, laser interferometry, and line broadening measurements.

Dr. Kayama is a member of the Brazilian Physical Society and the American Association of Physics Teachers.

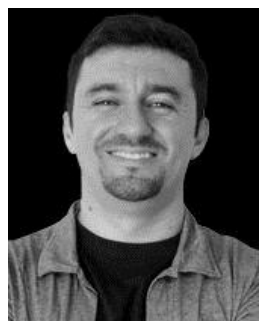

Lucas José da Silva received the bachelor's degree in physics and the master's degree in science from the Universidade Estadual Paulista, Guaratingueta, Brazil, in 2007 and 2015, respectively.

He was involved in the areas of synthesis, treatment, and the characterization of ceramic materials based on $\mathrm{TIO} 2$, using techniques of image processing. His current research interests include the generation of low-energy plasma for use in the treatment of surfaces of polymers and other materials and teaching.

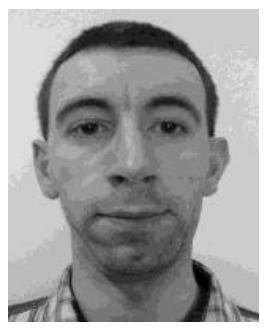

Vadym Prysiazhnyi received the master's diploma degree in applied physics from Taras Shevchenko National University, Kyiv, Ukraine, in 2006. In 2012, he defended his Ph.D. thesis in plasma physics at Masaryk University, Brno, Czech.

He successfully finished two post-doctoral fellowships in Czech Republic and Brazil, and was a Junior Researcher at CEPLANT, Brno. His current research interests include the design and characterization of plasma sources, its application in material treatments and deposition, and surface analysis techniques.

Konstantin G. Kostov, photograph and biography not available at the time of publication.

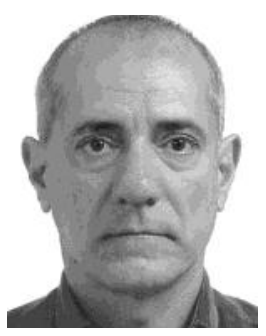

Mauricio Antonio Algatti was born in Birigüi, Brazil, in 1958. He received the bachelor's degree in physics, the M.S. degree in physical science and FIR laser physics, and the Ph.D. degree in physical science, ultrafast phenomena, and condensed matter physics from the Universidade Estadual de Campinas, Campinas, Brazil, in 1979, 1984, and 1990, respectively.

He was with MTA-KFKI, Budapest, Hungary. Since1992, he has been with the Plasma Group, Universidade Estadual Paulista (UNESP), Guaratinguetá, Brazil, developing research in plasma materials processing and synthesis for applications in biomaterials science and technology. Since 2011, he has been a Full Professor at the Physics and Chemistry Department, UNESP. 